\title{
Size-controlled chitosan nanoparticles prepared using ionotropic gelation
}

\author{
Setia Budi ${ }^{a, *}$, B. Asih Suliasih ${ }^{\mathrm{b}}$, Indah Rahmawati ${ }^{\mathrm{a}}$, Erdawati $^{\mathrm{a}}$ \\ a Department of Chemistry, Faculty of Mathematics and Science, Universitas Negeri Jakarta, \\ Jakarta Timur 13220 Indonesia \\ b Faculty of Pharmacy, Universitas Indonesia, Depok 16424 Indonesia
}

*Corresponding author, e-mail: setiabudi@unj.ac.id

Received 9 Jul 2019

Accepted $25 \mathrm{Jul} 2020$

\begin{abstract}
This work aimed at controlling the size of chitosan nanoparticles that were prepared by the ionotropic gelation method. Quantities of chitosan and sodium tripolyphosphate (STPP) were optimized to obtain a fine size and narrow size distribution of the nanoparticles. X-ray diffraction and selected area electron diffraction patterns indicated the existence of both crystalline and amorphous phases of chitosan. Particle size and size distribution were determined using transmission electron microscopy and dynamic laser scattering techniques. The size of the chitosan particles decreased with a reduction in STPP content due to a decrease in cross-link bonding and the formation of aggregate particles. Using this facile approach, the finest particles of chitosan were obtained in a range of 3-6 nm.
\end{abstract}

KEYWORDS: chitosan nanoparticles, ionotropic gelation method, size-controlled particles, narrow size distribution

\section{INTRODUCTION}

Chitosan is a biopolymer obtained through the deacetylation of the chitin polysaccharide found in the exoskeletons of crustaceans. This biopolymer recognized as a cationic polymer consisting of $\beta$-(1-4)-D-glucosamine and N-acetyl-D-glucosamine groups [1,2] exhibits biocompatible, biodegradable, non-toxic, antimicrobial, adsorptive, and environmentally friendly properties [3-6]. These properties have made chitosan a widely investigated and developed material for use in a variety of fields, including pharmaceuticals, agriculture, biomedicine, and the chemical industry [4, 7-9].

The properties of chitosan depend on its molecular weight, degree of polymerization, degree of deacetylation, and particle size $[3,10]$. In regards to particle size, nanoscale chitosan particles exhibit remarkable properties in its application such as high adsorption capacity [6], increase cellular uptake and drug release [11] and alleviate toxicological effect [12]. Previously, chitosan nanoparticles were synthesized using various methods, namely selfassembly [11,13], microemulsion [14], ultrafine milling [12] and ionotropic gelation $[2,15,16]$. The latter has been known as a simpler technique used for natural polymer nanoparticles preparation compared to others that require multi-experimental steps and use many harmful chemicals to assist preparation processes $[11,17]$, or are hard to control the chitosan particle size [18-20]. In the ionotropic gelation method, chitosan nanoparticles are prepared through the formation of cross-linked bonds between amino groups and the phosphate ions $\left(\mathrm{HPO}_{4}^{-}\right.$) from sodium tripolyphosphate (STPP). STPP is a non-toxic compound that forms a gel that has desirable properties [1]. This method can be performed without the need for organic solvents and does not require the use of high temperatures, and so molecular damage can be avoided $[5,10]$. However, the challenge that arises in nanoparticle preparation lies in the ability to obtain small-sized particles $[17,21]$ and to control their homogeneity $[6,10]$. It is well-known that, at the nanoscale level, particle size is among one of the main factors that determine the properties of a material. This paper reports on a successful approach for controlling the size and size distribution of chitosan particles. The resulting approach produced very fine particle sizes with a narrow size distribution.

\section{MATERIALS AND METHODS}

The chitosan nanoparticles were synthesized through an ionic gelation method using chitosan powder supplied by BATAN (The National Nuclear Agency of Indonesia) and STPP purchased from PT. Merck Indonesia. The amounts of chitosan and 
STPP used for each reaction were varied to observe the effects that different quantities had on the size and homogeneity of the chitosan nanoparticles. For this purpose, five different solution concentrations of each substance were prepared. STPP solutions were set with concentrations of $0.1,0.25,0.5$, 0.75 , and $1 \% \mathrm{w} / \mathrm{v}$, while the chitosan solutions each contained $0.2 \mathrm{~g}, 0.5 \mathrm{~g}, 1.0 \mathrm{~g}, 1.5 \mathrm{~g}$ and $2.0 \mathrm{~g}$. The dissolution of the chitosan was performed in $200 \mathrm{ml}$ of $1.0 \% \mathrm{v} / \mathrm{v}$ acetic acid by stirring at a speed of $1300 \mathrm{rpm}$ at $50^{\circ} \mathrm{C}$ for $2 \mathrm{~h}$. The solution was left for $24 \mathrm{~h}$ at room temperature. The STPP solution was then added gradually to the chitosan solution. This mixture was placed in a freezer for $24 \mathrm{~h}$, and afterwards, it was allowed to melt and then centrifuged at $6000 \mathrm{rpm}$ for $15 \mathrm{~min}$ to separate the water contained in the chitosan nanoparticles. Finally, the chitosan nanoparticles were freeze-dried for 4 days to obtain chitosan nanoparticle powder.

The prepared chitosan nanoparticles were characterized by a Shimadzu Prestige-21 Fourier transform infrared (FTIR) spectrometer to examine the occurrence of crosslinks between the phosphate ions of the STPP and the amino groups of the chitosan and to discover other functional chitosan groups. Xray diffraction (XRD) analysis was performed using an EMPYREAN PANalytical diffractometer. Particle size measurements were determined using a Malvern Zetasizer ZSP dynamic laser scattering particle size analyser (PSA). To further confirm the particle sizes, the samples were also analyzed by a transmission electron microscope (TEM). This TEM was also used to obtain a selected area electron diffraction (SAED) pattern to further confirm the crystalline phase of the nanoparticle chitosan.

\section{RESULTS AND DISCUSSION}

Fig. 1 shows the FTIR spectra of both the chitosan and chitosan nanoparticles. From the spectrum of the chitosan, the absorption band at wavenumber $3412 \mathrm{~cm}^{-1}$ is associated with the stretching of $-\mathrm{NH}_{2}$ and $-\mathrm{OH}[6,22,23]$, at $2924 \mathrm{~cm}^{-1}$ with $\mathrm{C}-\mathrm{H}$ stretching [23], and at $1745 \mathrm{~cm}^{-1}$ with the stretching of $\mathrm{C}=\mathrm{O}$ from a carbonyl group [6]. The identification of this carbonyl group indicates the existence of an acetyl group, which shows that the degree of deacetylation was less than $100 \%$. The degree of the deacetylation of the chitosan in this study was $94.50 \%$, indicating its purity and related properties [6]. The other absorption bands observed at wavenumber of $1639 \mathrm{~cm}^{-1}, 1463 \mathrm{~cm}^{-1}, 1317 \mathrm{~cm}^{-1}$, $1159 \mathrm{~cm}^{-1}$ and $890 \mathrm{~cm}^{-1}$ are associated to a primary
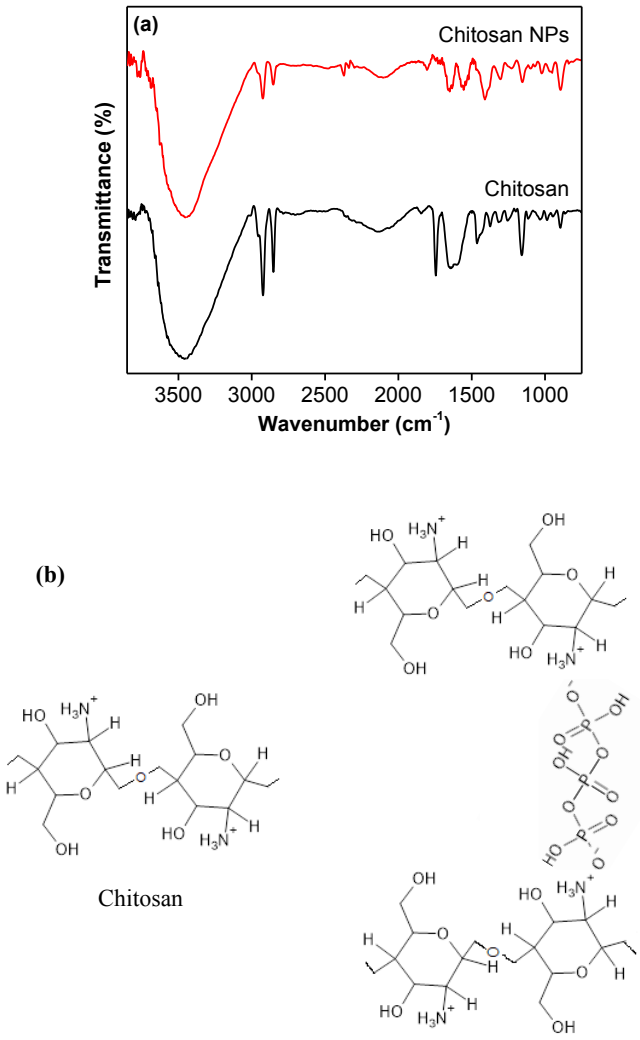

Chitosan nanoparticles

Fig. 1 (a) FTIR spectra of the raw chitosan and the prepared chitosan nanoparticles (NPs). (b) The structure of raw chitosan and ionic cross-linking configuration in chitosan nanoparticles.

amide bending group $-\mathrm{C}=\mathrm{O}-[10,22]$, $\mathrm{CH}$ group bending [6], stretching of tertiary amides $[6,10]$, the presence of amino $\mathrm{NH}_{2}$, and $\mathrm{C}-\mathrm{H}$ [22], respectively.

In the FTIR spectrum of the chitosan nanoparticles, several absorption bands at 3425, 2924, 1303 and $890 \mathrm{~cm}^{-1}$ were found to be slightly shifted as compared to the ones observed in the chitosan powder. In addition, the band at wavenumber $1745 \mathrm{~cm}^{-1}$ disappeared due to the formation of a cross-link between the phosphate ion from the STPP and the amino group from the chitosan [6]. A band at wavenumber $1115 \mathrm{~cm}^{-1}$ also shows a cross bond between a negative phosphate group from the STPP with a positively charged amino group from the chitosan, which is in this case, the oxygen of the phosphate group acting as a bridge between the nitrogen from an amino group and the phosphorous from a phosphate group $[2,10,24]$. The formation of a cross-link bond was also confirmed 


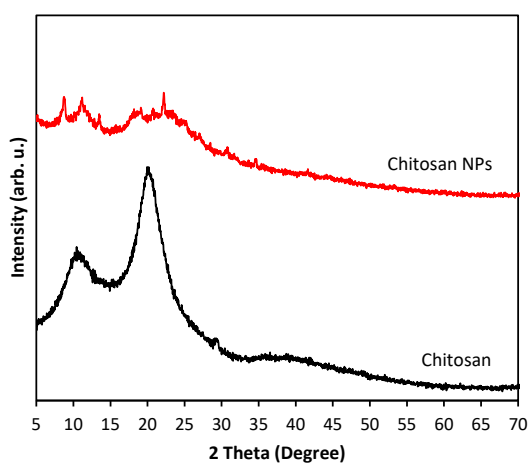

Fig. 2 XRD patterns of the raw chitosan and prepared chitosan Nps.

by the appearance of two new absorption bands at wavenumber of $1651 \mathrm{~cm}^{-1}$ and $1554 \mathrm{~cm}^{-1}$, which are associated with $-\mathrm{CONH}_{2}$ and $\mathrm{N}-\mathrm{H}$, respectively. These two bands also indicate an increase in the inter- and intra-molecular forces in the chitosan nanoparticles $[1,8,22,24]$. One other peak observed at wave number $1099 \mathrm{~cm}^{-1}$ is associated with $\mathrm{C}-\mathrm{N}$ stretching [22].

The XRD diffraction patterns of the chitosan and chitosan nanoparticles are shown in Fig. 2 Diffraction peaks at $2 \theta$ of $10.18^{\circ}$ and $20.26^{\circ}$ were observed in the chitosan powder, each of which is a reflection of the (020) hydrated crystalline structure and (110) crystalline structure of anhydrous $\alpha$ chitin [25]. The two diffraction peaks were also observed in the chitosan nanoparticles diffraction pattern. This indicates the presence of a crystalline phase in the synthesized chitosan nanoparticles. The SAED pattern taken using TEM (Fig. 4c) further confirms this crystalline phase. Meanwhile, the amorphous chitosan phase was detected with the appearance of broad peaks at $2 \theta$ of $22^{\circ}$. By comparing the XRD peaks, it can be seen clearly that the peaks in intensity of the chitosan nanoparticles are lower than that of the chitosan powder. Besides being due to the refining of particle size, the decrease in peak intensity also indicates the occurrence of the cross-linking between chitosan and STPP, which results in modifications to the molecular arrangement of the crystal lattices [24, 26, 27].

Furthermore, to determine the influence of the chitosan and STPP compositions on particle size, the size of the chitosan nanoparticle was measured using the PSA. Fig. 3a shows the particle size distribution of the chitosan nanoparticles that was obtained by varying the content of chitosan with a fixed STPP concentration at $0.5 \% \mathrm{w} / \mathrm{v}$. Based
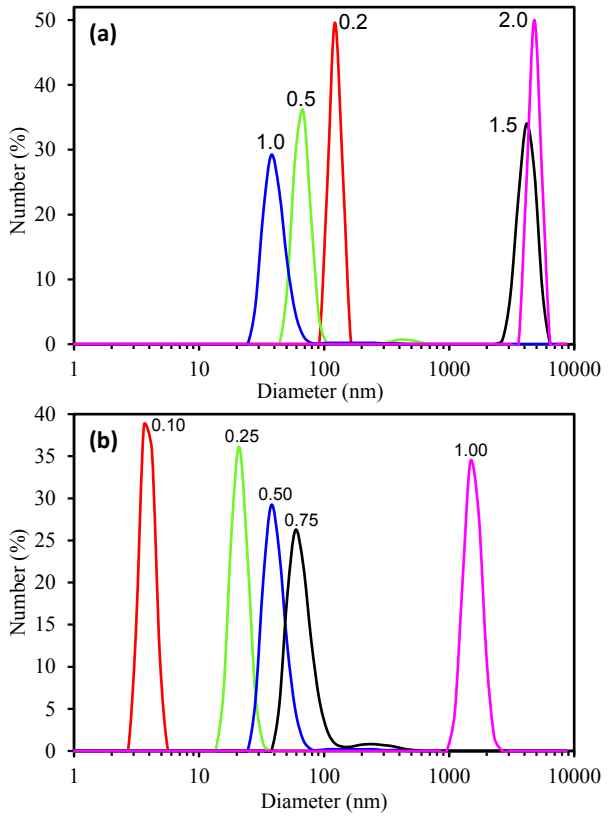

Fig. 3 Particle size distribution of the chitosan nanoparticles prepared by varying (a) the mass of chitosan $(0.2$, $0.5,1.0,1.5$ and $2.0 \mathrm{~g})$ and (b) the content of STPP (0.10, $0.25,0.50,0.75$ and $1.00 \% \mathrm{w} / \mathrm{v}$ ).

on the five different chitosan amounts studied in this work, the finest particle size was obtained with $1.0 \mathrm{~g}$ of chitosan. The curve shows that the prepared chitosan particles were in the range of 20$70 \mathrm{~nm}$. This measurement result agrees with the TEM micrographs shown in Fig. 4a-b. From the PSA curve, it can also be clearly seen that there is a narrow size distribution based on a polydispersity index (PdI) of 0.3, indicating a homogeneous size of the particles. PdI is a value that describes the distribution of particle size and is calculated using the following equation: $\operatorname{PdI}=(\sigma / d)^{2}$, where $\sigma$ is standard deviation and $d$ is diameter.

Homogeneous particles are indicated by a PdI value between 0 and 0.5 , while values greater than 0.5 represent heterogeneous particle sizes $[28,29]$. At the lower chitosan content levels, 0.2 and $0.5 \mathrm{~g}$, there was no significant change in particle size as compared to that obtained from the $1.0 \mathrm{~g}$ composition. However, very large size particles $(>2000 \mathrm{~nm})$ were observed in samples obtained with higher chitosan content levels, namely 1.5 and $2.0 \mathrm{~g}$. These results indicate an increase in cross-linking formation and particle aggregation with higher chitosan content, thus increasing particle size [30].

Fig. 3b shows the particle size distribution of the chitosan nanoparticles prepared with different 

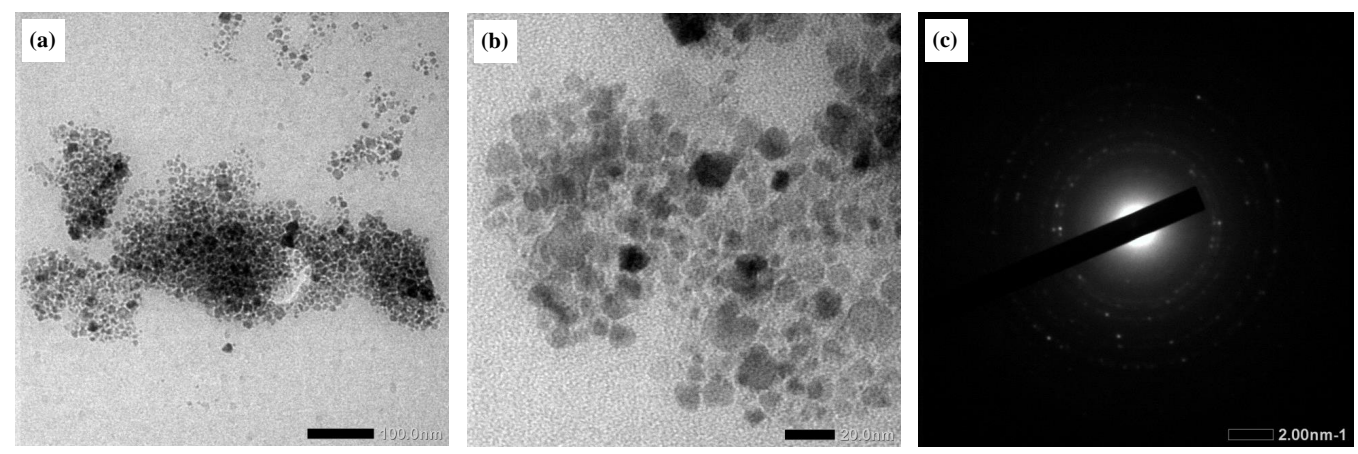

Fig. 4 (a,b) TEM micrographs and (c) SAED pattern of nanoparticle chitosan prepared with a method containing $1.0 \mathrm{~g}$ of chitosan and $0.5 \% \mathrm{w} / \mathrm{v}$ of STPP.

STPP concentrations using a solution containing $1.0 \mathrm{~g}$ of chitosan. The distribution curves show that the particle size became smaller with decreasing concentrations of STPP. In this study, the smallest particles sizes were in the range of 3-6 nm and were obtained with the lowest concentration of STPP, namely $0.1 \% \mathrm{w} / \mathrm{v}$. At this concentration, a very narrow size distribution is represented by the close values of Dv10, Dv50, and Dv90 (Dv = volume based distribution) for 3.72, 4.57 and 5.55, respectively. The decrease in STPP concentration has a role in reducing inter- and intramolecular bonds, and so this may reduce the occurrence of the formation of larger particles. Since the chitosan nanoparticles formed through cross-linking formation between phosphate ion $\left(\mathrm{HPO}_{4}^{-}\right)$from STPP structure and chitosan protonated amino group, low concentration of STPP prevented the particles from growing. In addition, a lower concentration of STPP may also minimize the aggregation that contributes to the formation of larger particles. Hence low concentration produced finer chitosan nanoparticles compared to those obtained from high concentration of STPP $[2,8,30,31]$. This result shows that the applied approach was beneficial to produce very fine nano-sized particles $(3-6 \mathrm{~nm}$ ) compared to those previously reported that range from 105-615 nm prepared by ionotropic gelation method [17,21], and from $210 \mathrm{~nm}$ to micro-sized particles prepared by other methods $[19,20,23]$.

\section{CONCLUSION}

The chitosan nanoparticles were successfully synthesized by varying chitosan and STPP composition using an ionotropic gelation method. To produce very small chitosan particles, the chitosan and STPP compositions were varied. The results show that the particle size and their distribution were controlled through the adjustment of chitosan and STPP compositions. Through this approach, fine particles with a very narrow size distribution range of 3-6 $\mathrm{nm}$ were successfully prepared. The quantity of both chitosan and STPP have been shown to play a very important role in controlling particle size. The optimum amount of chitosan to obtain small size particles was 1.0 g. Meanwhile, a lower concentration of STPP resulted in finer chitosan particles due to a decline in the cross-link bonding is responsible for particle formation as well as particle aggregation.

Acknowledgements: This work was financially supported by Universitas Negeri Jakarta.

\section{REFERENCES}

1. Antoniou J, Liu F, Majeed H, Qi J, Yokoyama W, Zhong F (2015) Physicochemical and morphological properties of size-controlled chitosantripolyphosphate nanoparticles. Colloids Surf A Physicochem Eng Asp 465, 137-146.

2. Ibrahim HM, El-Bisi MK, Taha GM, El-Alfy EA (2015) Chitosan nanoparticles loaded antibiotics as drug delivery biomaterial. J Appl Pharm Sci 5, 85-90.

3. Caro León FJ, Lizardi-Mendoza J, Argüelles-Monal W, Carvajal-Millan E, López Franco YL, Goycoolea FM (2017) Supercritical $\mathrm{CO}_{2}$ dried chitosan nanoparticles: production and characterization. RSC Adv 7, 30879-30885.

4. Nguyen TV, Nguyen TTH, Wang SL, Vo TPK, Nguyen AD (2017) Preparation of chitosan nanoparticles by TPP ionic gelation combined with spray drying, and the antibacterial activity of chitosan nanoparticles and a chitosan nanoparticle-amoxicillin complex. Res Chem Intermed 43, 3527-3537.

5. Rampino A, Borgogna M, Blasi P, Bellich B, Cesáro A (2013) Chitosan nanoparticles: Preparation, size evolution and stability. Int J Pharm 455, 219-228.

6. Sivakami MS, Gomathi T, Venkatesan J, Jeong HS, Kim SK, Sudha PN (2013) Preparation and character- 
ization of nano chitosan for treatment wastewaters. Int J Biol Macromol 57, 204-212.

7. Sreekumar S, Goycoolea FM, Moerschbacher BM, Rivera-Rodriguez GR (2018) Parameters influencing the size of chitosan-TPP nano- and microparticles. Sci Rep 8, ID 4695.

8. Vimal S, Abdul Majeed S, Taju G, Nambi KSN, Sundar Raj N, Madan N, Farook MA, Rajkumar T, et al (2013) Chitosan tripolyphosphate (CS/TPP) nanoparticles: Preparation, characterization and application for gene delivery in shrimp. Acta Trop 128, 486-493.

9. Zhu L, Fan Z-Q, Shi X-Q, Wang N, Bo Y-Y, Guo HE (2020) A novel silkworm pupae carboxymethyl chitosan inhibits mouse L929 fibroblast proliferation. Sci Asia 46, 30-36.

10. Vaezifar S, Razavi S, Golozar MA, Karbasi S, Morshed M, Kamali M (2013) Effects of some parameters on particle size distribution of chitosan nanoparticles prepared by ionic gelation method. J Clust Sci 24, 891-903.

11. Yu Z, Ma L, Ye S, Li G, Zhang M (2020) Construction of an environmentally friendly octenylsuccinic anhydride modified $\mathrm{pH}$-sensitive chitosan nanoparticle drug delivery system to alleviate inflammation and oxidative stress. Carbohydr Polym 236, ID 115972.

12. Naiel MAE, Ismael NEM, Abd El-hameed SAA, Amer MS (2020) The antioxidative and immunity roles of chitosan nanoparticle and vitamin C-supplemented diets against imidacloprid toxicity on Oreochromis niloticus. Aquaculture 523, ID 735219.

13. Quiñones JP, Peniche H, Peniche C (2018) Chitosan based self-assembled nanoparticles in drug delivery. Polymers 10, ID 235.

14. Martins AF, de Oliveira DM, Pereira AGB, Rubira AF, Muniz EC (2012) Chitosan/TPP microparticles obtained by microemulsion method applied in controlled release of heparin. Int J Biol Macromol 51, 1127-1133.

15. Zahiri M, et al (2020) Encapsulation of curcumin loaded chitosan nanoparticle within poly $(\varepsilon$ caprolactone) and gelatin fiber mat for wound healing and layered dermal reconstitution. Int $J$ Biol Macromol 153, 1241-1250.

16. Kamat V, Bodas D, Paknikar K (2016) Chitosan nanoparticles synthesis caught in action using microdroplet reactions. Sci Rep 6, ID 22260.

17. Qu G, Yao Z, Zhang C, Wu X, Ping Q (2009) PEG conjugated N-octyl-O-sulfate chitosan micelles for delivery of paclitaxel: In vitro characterization and in vivo evaluation. Eur J Pharm Sci 37, 98-105.

18. Rahmi, Lelifajri, Ayu ASW (2020) Preparation of magnetic chitosan microspheres using iron sand particles prepared by ball milling method. IOP Conf Ser: Mater Sci Eng 796, ID 012043.
19. Qiu W, Vakili M, Cagnetta G, Huang J, Yu G (2020) Effect of high energy ball milling on organic pollutant adsorption properties of chitosan. Int J Biol Macromol 148, 543-549.

20. Wani TA, Masoodi FA, Akhter R, Sofi FA (2020) Techno-functional characterization of chitosan nanoparticles prepared through planetary ball milling. Int J Biol Macromol 154, 166-172.

21. Koukaras EN, Papadimitriou SA, Bikiaris DN, Froudakis GE (2012) Insight on the formation of chitosan nanoparticles through ionotropic gelation with tripolyphosphate. Mol Pharm 9, 2856-2862.

22. Manikandan A, Sathiyabama M (2016) Preparation of chitosan nanoparticles and its effect on detached rice leaves infected with Pyricularia grisea. Int J Biol Macromol 84, 58-61.

23. Mukhopadhyay P, Sarkar K, Chakraborty M, Bhattacharya S, Mishra R, Kundu PP (2013) Oral insulin delivery by self-assembled chitosan nanoparticles: In vitro and in vivo studies in diabetic animal model. Mater Sci Eng C 33, 376-382.

24. Bhumkar DR, Pokharkar VB (2006) Studies on effect of $\mathrm{pH}$ on cross-linking of chitosan with sodium tripolyphosphate: A technical note. AAPS Pharm Sci Tech 7, E138-E143.

25. Guirguis O, Abdelzaher N, El-Bassyouni G, Moselhey M (2018) Structural, thermal and optical modifications of chitosan due to UV-ozone irradiation. Egypt J Chem 61, 447-460.

26. Shah S, Pal A, Kaushik VK, Devi S (2009) Preparation and characterization of venlafaxine hydrochlorideloaded chitosan nanoparticles and in vitro release of drug. J Appl Polym Sci 112, 2876-2887.

27. Yao Q, Liu W, Gou X, Guo X, Yan J, Song Q, Chen F, Zhao Q, et al (2013) Preparation, characterization, and cytotoxicity of various chitosan nanoparticles. $J$ Nanomater 2013, ID 183871.

28. Anbu AS, et al (2016) Synthesis of bioactive chemicals cross-linked sodium tripolyphosphate (TPP): chitosan nanoparticles for enhanced cytotoxic activity against human ovarian cancer cell line (PA-1). $J$ Nanomed Nanotechnol 7, ID 418.

29. Huang X, Li B, Zhang H, Hussain I, Liang L, Tan B (2011) Nanoscale facile preparation of size-controlled gold nanoparticles using versatile and end-functionalized thioether polymer ligands. Nanoscale 3, 1600-1607.

30. Ali SW, Joshi M, Rajendran S (2011) Synthesis and characterization of chitosan nanoparticles with enhanced antimicrobial activity. Int J Nanosci 10, 979-984.

31. Mudhakir D, Wibisono C, Rachmawati H (2014) Encapsulation of risperidone into chitosan-based nanocarrier via ionic binding interaction. Procedia Chem 13, 92-100. 